\title{
Distributive Analysis of Head and Neck Swellings with Their Cytopathological Correlation
}

\author{
${ }^{1}$ Apoorva K Pandey, ${ }^{2}$ Aparna Bhardwaj, ${ }^{3}$ Tripti Maithani, ${ }^{4}$ Sanjeev Kishore, ${ }^{5}$ VP Singh
}

\begin{abstract}
Aim: Palpable masses in the head and neck region can arise from various structures, such as lymph node, thyroid gland, major and minor salivary glands, soft tissues, blood vessels, and neural structures. Fine needle aspiration cytology (FNAC) is a simple, quick, inexpensive first-line method to evaluate such swellings and thus confirm the diagnosis. This study correlates aspiration cytology as an indispensable tool in diagnosing various head and neck swellings and their prevalence with respect to age, sex, site of origin, and nature of those masses.
\end{abstract}

Materials and methods: This study was done from January 2007 to December 2013 in the Department of Otorhinolaryngology and Department of Pathology. All the patients presenting with various head and neck swellings underwent FNAC, and the results were correlated with anatomical sites and frequency of occurrence and categorized into various clinicopathological groups.

Results: This study included 1,272 cases who were evaluated by FNAC for head and neck swellings. In this study, 32 patients presented with frank abscesses, 36 cases with submandibular region swellings, seven cases with submental swellings, 70 cases with various swellings in the neck and postauricular area, 47 cases with various facial and scalp swellings, two cases from oral cavity, 74 cases with parotid masses, 680 cases with lymphadenopathy, and 324 cases with thyroid lesions.

Conclusion: Fine needle aspiration cytology is a useful, reliable, cost-effective, and valuable diagnostic tool for the initial evaluation and diagnostic categorization of all superficial and deep-seated lesions in the head and neck region as it is a fairly sensitive and specific procedure in addition to being simple, rapid, accurate, and without any significant complications and above all without affecting the underlying tissue morphology altogether.

Keywords: Cervical lymphadenopathy, Fine needle aspiration cytology, Lymphoma, Parotid swellings, Thyroid, Tuberculosis.

How to cite this article: Pandey AK, Bhardwaj A, Maithani T, Kishore S, Singh VP. Distributive Analysis of Head and Neck Swellings with Their Cytopathological Correlation. Int J Otorhinolaryngol Clin 2016;8(3):89-96.

\footnotetext{
${ }^{1,2}$ Associate Professor, ${ }^{3}$ Professor, ${ }^{4,5}$ Professor and Head

1,3,5 Department of ENT, Shri Guru Ram Rai Institute of Medical and Health Sciences, Dehradun, Uttarakhand, India

${ }^{2,4}$ Department of Pathology, Shri Guru Ram Rai Institute of Medical and Health Sciences, Dehradun, Uttarakhand, India

Corresponding Author: Apoorva K Pandey, Associate Professor, Department of ENT, Shri Guru Ram Rai Institute of Medical and Health Sciences, Dehradun, Uttarakhand, India Phone: +91-9411324477, e-mail: pande.apoorva@gmail.com
}

\section{Source of support: Nil}

Conflict of interest: None

\section{INTRODUCTION}

Encountering patients with a head and neck mass is frequently met with difficulty in routine ear, nose, and throat (ENT) practice, and the intricate anatomy of this important region often results in puzzling circumstances in diagnosing these lesions. The patient's age, location, size, onset, and duration of a swelling are important clues for making a specific differential diagnosis. The common pathologies usually are from enlargement of lymph nodes (lymphoproliferative disorder, inflammatory process, or infiltration by metastatic malignant cells), thyroid gland (goiter, thyroiditis, benign and malignant tumors), salivary glands (sialadenitis, cysts, benign and malignant tumors), and various cysts like thyroglossal and branchial cysts, epidermoid and dermoid cysts, and lipomas. Neoplasia makes a significant differential diagnostic consideration because neck mass is most often the first and sole representation of this process. It also helps in detecting recurrences or emergence of new tumors after treatment, obviating the need of any surgical intervention in most cases. The history of assessing various organs by aspiration technique for diagnostic and therapeutic indications dates back to almost one and a half century. James Paget, in 1853, described cells aspirated from breast malignancies and Grieg and Gray, in 1904, identified trypanosomes in aspirated specimens of lymph glands. This technique was later used by Guthrie, in 1921, to examine malignant lymphomas and also by Martin and Ellis to study various swellings. Following the same footsteps, Stewart, in 1933, published a series on the diagnosis of 2500 tumors. ${ }^{1}$ Swellings in the head and neck region include inflammatory, cystic, congenital, various specific and nonspecific lesions, and benign and malignant pathologies, and their timely diagnosis furnishes the best chance of effective treatment.

The aims of our study were as follows:

- Classifying various head and neck swellings according to anatomic areas

- Correlating those swellings with most common clinical complaints

- Assessing prevalence, female or male predilection, and age-related distribution 
- Differentiating them between cystic, inflammatory, benign, and malignant groups

- Typifying the benign and malignant entities

- Reviewing the pitfalls in cytological diagnosis with respect to review of literature

\section{MATERIALS AND METHODS}

This study was conducted at the tertiary care centre of Dehradun, Uttarakhand, from January 2007 to December 2013. Each patient was thoroughly examined in the Department of ENT, and details regarding history, clinical examination, and probable diagnosis were noted, and data regarding age, sex, anatomic region, and presenting complaints were correlated. All head and neck masses were further referred to the Department of Pathology for cytological examination. Fine needle aspiration cytology (FNAC) was done with the help of $10 \mathrm{~mL}$ disposable syringe with an attached 21-gauge needle. A few of the aspirations, particularly from smaller and difficult to palpate lesions, were guided. Slides were air-dried and stained by the May Grünwald Giemsa stain. Rest of the slides were fixed in methanol and stained with Papanicolaou and Hematoxylin and eosin (H\&E) stains. In the case of lymphadenopathy, where suspicion of tubercular pathology was there or aspiration yielded cheesy/caseous/necrotic material, Ziehl Neelsen (ZN) stain for acid-fast bacilli was done. An average of three passes was made in large cystic swellings, particularly in parotid and thyroid lesions. This study was approved by the institutional ethics committee.

\section{OBSERVATION}

Total number of cases included in this study was 1,272, and among them, 630 (49.53\%) were males and 642 $(50.47 \%)$ were females, female to male ratio being 1.01:1. The youngest patient in this series was 16 days old and the

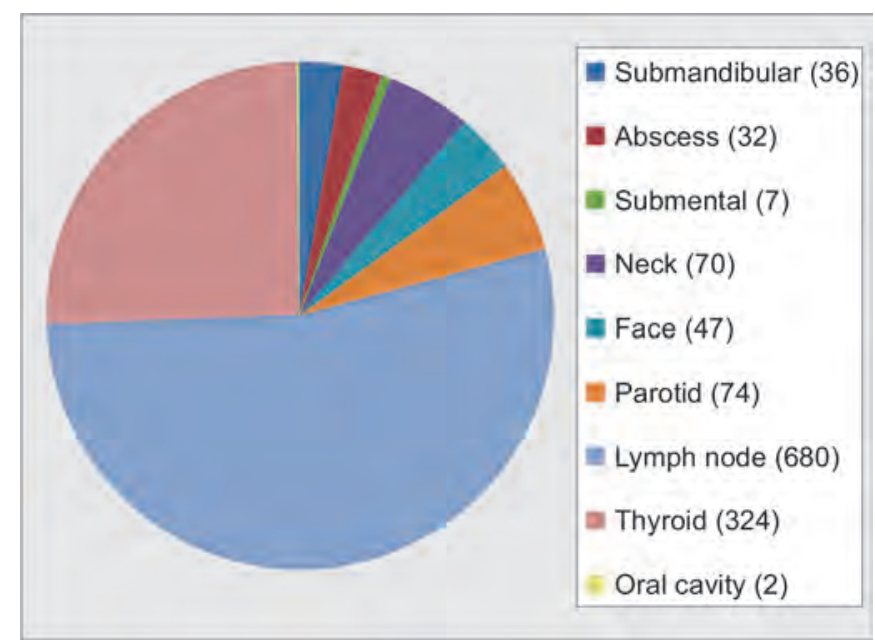

Graph 1: Distribution of total head and neck swellings $(n=1,272)$ oldest was 90 years old, and the mean age was 34.24 years. Most of the cases aspirated were from lymph nodes 680 $(53.46 \%)$, followed by 324 cases $(25.47 \%)$ from thyroid gland, 74 cases $(5.82 \%)$ from parotid region, 36 cases $(2.83 \%)$ from submandibular region, and there were total 61 cases $(4.8 \%)$ whose reports were inconclusive (Graph 1 ).

Out of the total of 1,272 cases, 32 cases presented with frank abscess features clinically, which was later confirmed on aspiration. The most common complaints were pain, enlargement, and fever in occasional cases. There were 21 abscesses of tubercular origin, and 11 cases were of acute suppurative/pyogenic origin. In this group, there were 20 males and 12 females. Abscesses were in the right side in 22 cases, 1 in midline, and 9 on the left side. The eldest patient was a 70-year-old male, while the youngest was a 16-day-old male. All supraclavicular (4 cases) and submandibular (1 case) abscesses were tubercular in origin (Graph 2).

A total of 36 cases had swellings in the submandibular region; of those, 22 cases presented with right-sided swellings and 14 had left-sided swellings. There were 13 females and 23 males in this group. The eldest patient was 76 years old, while the youngest was 7 years old. The majority of cases had sialadenitis 18 (50\%) followed by pleomorphic adenoma 5 (13.9\%), and retention cyst $5(13.9 \%)$. Most common clinical complaints were enlargement, pain in the submandibular area, pain on swallowing, and fever. The other pathologies observed were as follows: Sialadenosis 2 (5.5\%); one case $(2.8 \%)$ each of mucoepidermoid carcinoma, lipoma, epidermoid cyst; and inconclusive 3 (8.3\%) (Graph 3).

There were a total of seven patients who presented with submental swellings. There were six males and a single female. The oldest patient was 60 years old, while the youngest was 5 years. There were three cases (42.9\%) each of acute suppurative lesion and mucocele and one $(14.2 \%)$ benign cyst (Table 1$)$. Most common

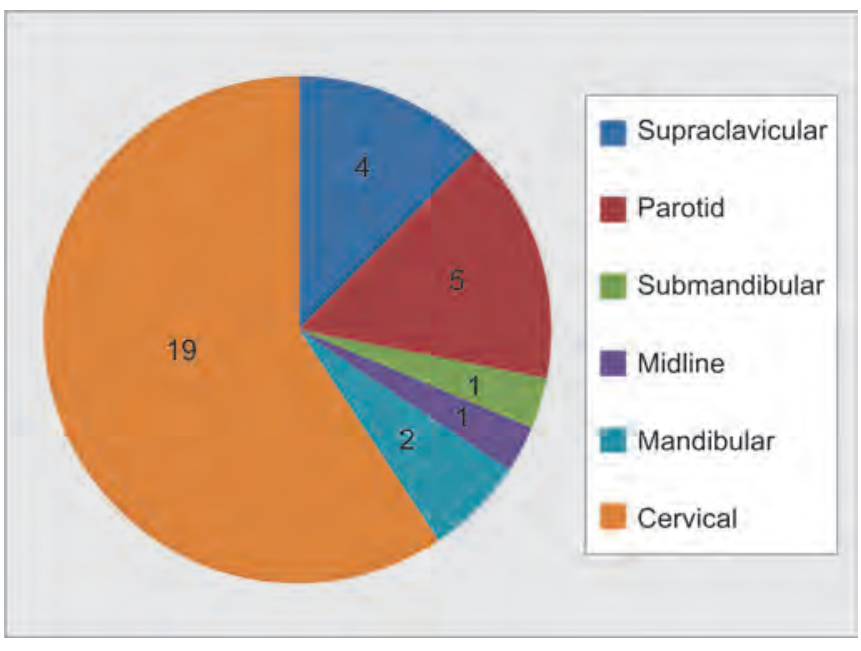

Graph 2: Distribution of various abscesses $(n=32)$ 


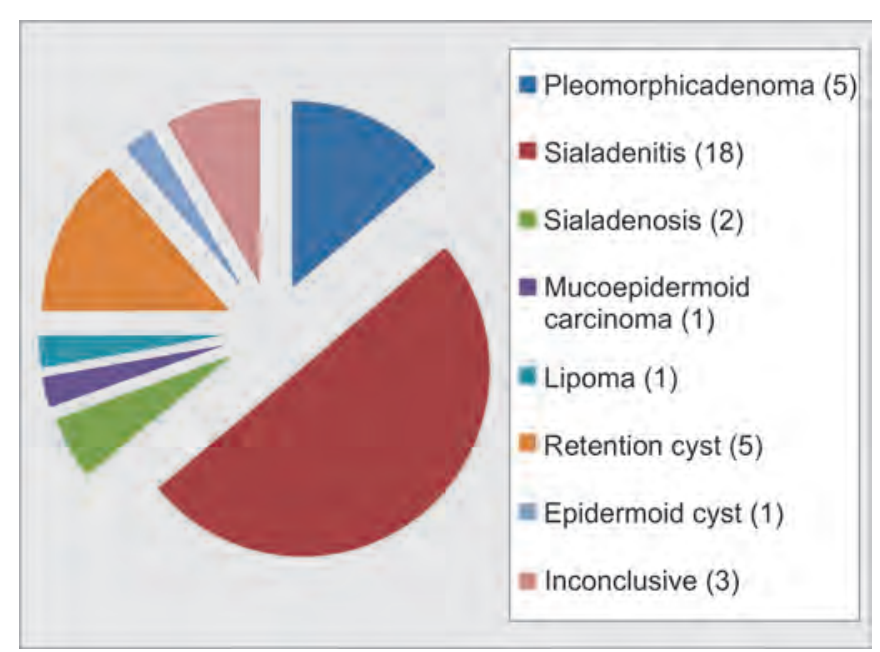

Graph 3: Distribution of submandibular swellings $(n=36)$

Table 1: Distribution of submental swellings

\begin{tabular}{llll}
\hline Sl. no. & Cytological diagnosis & Number of cases & Percentage \\
\hline 1 & Acute suppurative & 3 & 42.9 \\
& lesion & & \\
2 & Mucocele & 3 & 42.9 \\
3 & Benign cyst & 1 & 14.2 \\
\hline & Total & 7 & \\
\hline
\end{tabular}

clinical complaints were enlargement and pain in the submental area.

There were a total of 70 cases of various neck swellings, and of those 40 were males and 30 were females. Most common clinical complaint was enlargement. The oldest case was 71 years old, while the youngest was 3 years of age. Majority of cases were epidermoid cyst 17 (24.3\%), lipoma $15(21.4 \%)$, thyroglossal cyst $8(11.5 \%)$, and branchial cyst 7 (10\%) (Graph 4). Among branchial cysts, four were right-sided and 3 were on the left side.

Total cases having swellings in face and scalp were 47. There were 25 males and 22 females. The oldest patient was 83 years old, and the youngest was 1 year old. Most common clinical complaints were enlargement and pain, particularly in acute inflammatory cases. Most common pathology observed was epidermoid cyst $14(29.79 \%)$ followed by acute suppurative lesion 7 (14.89\%), granulomatous inflammation and lipoma 4 each $(8.51 \%)$, and lymphangioma and benign vascular lesion 2 each (4.26\%). Malignant lesions observed were basal cell carcinoma (BCC) 1 case and non-Hodgkin's lymphoma (NHL) one case. Eleven cases were inconclusive (Table 2).

Among 74 patients having swellings in the parotid area, 41 were males and 33 females. Youngest patient in this group was 1.5 years, whereas the oldest was 82 years. There were 38 lesions on the right side, 35 on the left side, and one case presented with bilateral parotid swelling. Most common clinical complaint was enlargement, followed by pain and fever. The commonest pathology

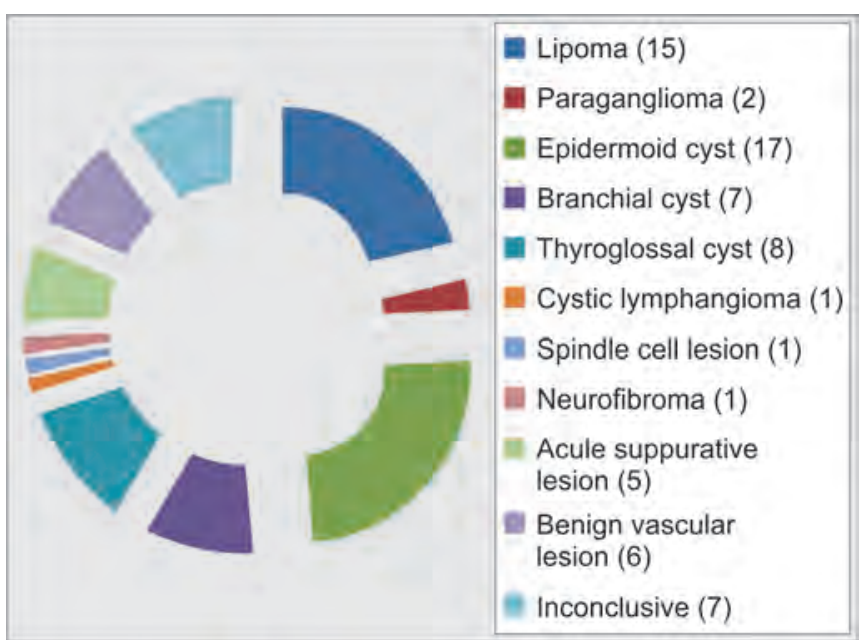

Graph 4: Distribution of total neck and postauricular swellings $(n=70)$

observed was pleomorphic adenoma 29 (39.2\%), followed by sialadenitis $14(18.9 \%)$, Warthin's tumor and sialadenosis $8(10.9 \%)$ each), and mucoepidermoid carcinoma and squamous cell carcinoma $2(2.8 \%)$ each) (Graph 5). Hemorrhages, infection, injury to facial nerve, seeding of tumor cells, or any other complications were not noticed.

Out of 680 lymphadenopathy cases, 434 patients (63.9\%) had involvement of deep jugular group of lymph

Table 2: Distribution of various facial swellings

\begin{tabular}{lll}
\hline Cytological diagnosis & Number of patients & Percentage \\
\hline Lymphangioma & 2 & 4.25 \\
Granulomatous inflammation & 4 & 8.51 \\
Epidermoid cyst & 14 & 29.79 \\
Sebaceous cyst & 1 & 2.13 \\
Acute suppurative lesion & 7 & 14.89 \\
Lipoma & 4 & 8.51 \\
Benign vascular lesion & 2 & 4.26 \\
BCC & 1 & 2.13 \\
NHL & 1 & 2.13 \\
Inconclusive & 11 & 23.4 \\
\hline Total & 47 & \\
\hline
\end{tabular}

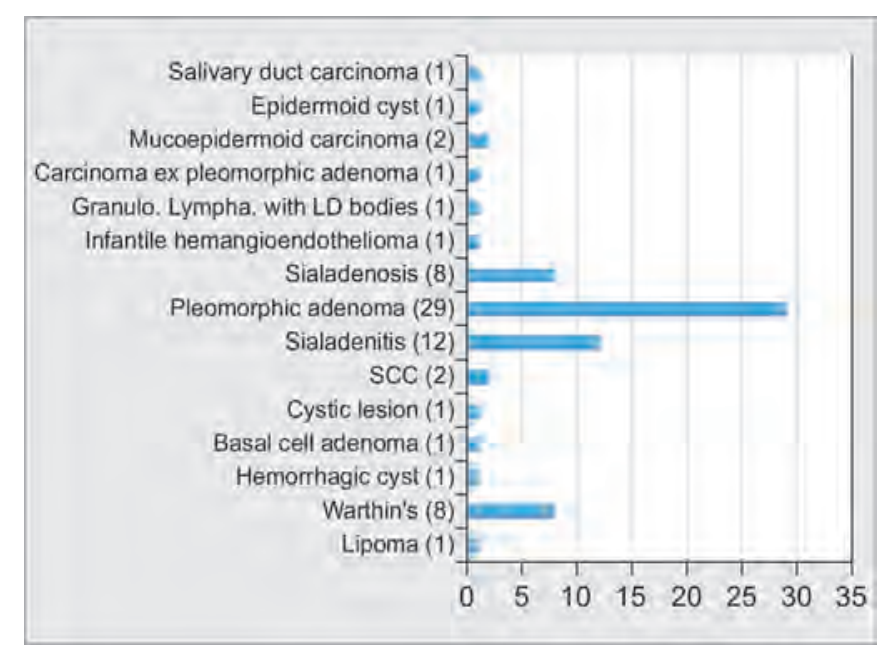

Graph 5: Distribution of various pathologies in parotid region $(n=74)$ 
Table 3: Distribution of various lymph node groups

\begin{tabular}{llll}
\hline SI. no. & Lymph node site & Number & Percentage \\
\hline 1 & $\begin{array}{l}\text { Deep jugular lymph } \\
\text { node }\end{array}$ & R-225, L-176, B/L-33 & 63.8 \\
2 & Postauricular & R-11, L-3, B/L-3 & 2.5 \\
3 & Submandibular & R-30, L-17, B/L-1 & 7.1 \\
4 & Submental & 21 & 3.1 \\
5 & Supraclavicular & R-29, L-34 & 9.3 \\
6 & Posterior triangle & R-26, L-12, B/L-2 & 5.9 \\
7 & Multiple lymph nodes & 37 & 5.4 \\
8 & Preauricular & R-8, L-1 & 1.3 \\
9 & Occipital & R-7, L-4 & 1.5 \\
\hline & Total & 680 &
\end{tabular}

nodes; supraclavicular group of lymph nodes was the second most common affected site $(9.3 \%)$ in the neck (Table 3). Most of the cases presented with unilateral cervical lymph node involvement. There was male predominance 380 (55.9\%) observed, and male to female ratio was 1.27:1. Fine needle aspiration cytology was found to be inconclusive in 24 cases (3.5\%). Most common clinical complaint was enlargement associated with pain and fever in acute inflammatory and tubercular cases. Significant complaints like weight loss and loss of appetite were observed in elderly patients. The most common cause of enlarged neck nodes was reactive hyperplasia in 263 cases (38.7\%). Second most common cause was tuberculosis, which accounted for 175 cases $(25.7 \%)$. This was followed in frequency by granulomatous type in 92 cases $(13.5 \%)$, metastatic lymphadenopathy in 69 cases $(10.2 \%)$, and acute suppuration in 34 cases $(5 \%)$. There were 16 cases $(2.3 \%)$ of NHL and only five cases $(0.7 \%)$ of Hodgkin's lymphoma (HL) (Graph 6). The comparison between reactive, tuberculous, granulomatous, and metastatic lymphadenopathy in various age groups is given in Graph 7. Highest incidence of malignancy was observed in the 61 to 70 years age group. Reactive hyperplasia was most commonly seen in $<10$ years age group, tuberculous

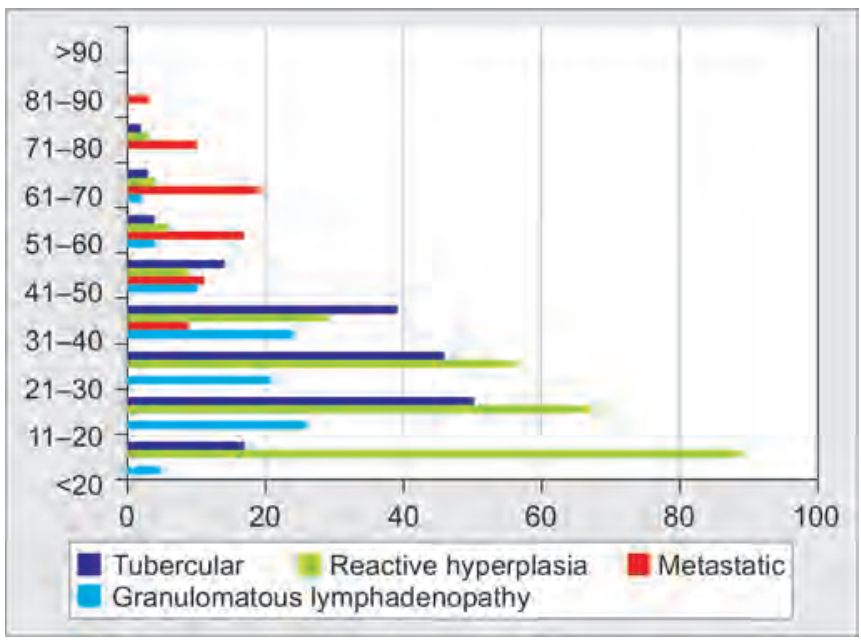

Graph 7: Distribution of lymphadenopathies among various age groups

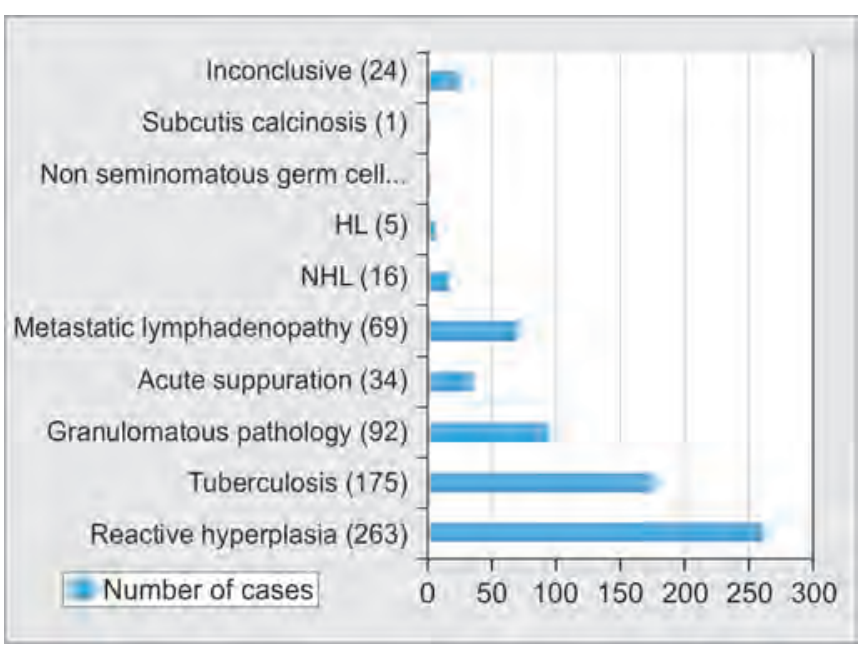

Graph 6: Cytologic distribution of various lymphadenopathies $(n=680)$

type in 11 to 20 years age group, and granulomatous type in 11 to 20 years age group.

Total number of patients having a thyroid swelling was 324, and females (231) outnumbered males (93), the female to male ratio being 2.48:1. Most common clinical complaint was enlargement, whereas voice change and difficulty in swallowing were noted in markedly enlarged swellings and fever in acute inflammatory cases. The commonest lesion observed in thyroid gland was colloid goiter $209(64.5 \%)$ succeeded by thyroiditis $59(18.2 \%)$. In thyroiditis group (59 cases), lymphocytic variant was the most common 49 (83\%) followed by granulomatous $8(13.6 \%)$ and acute suppurative type $2(3.4 \%)$. Among the 19 malignant lesions (5.9\%), follicular neoplasm $10(52.6 \%)$ was the most common pathology observed followed by papillary carcinoma $4(21.1 \%)$, Hurthle cell neoplasm $3(15.8 \%)$, and least was medullary carcinoma $2(10.5 \%)$. Other pathologies observed were as follows: Hyperplastic nodule 14 (4.3\%), Grave's disease $7(2.2 \%)$, NHL $1(0.3 \%)$, and 15 cases $(4.6 \%)$ were inconclusive (Graph 8).

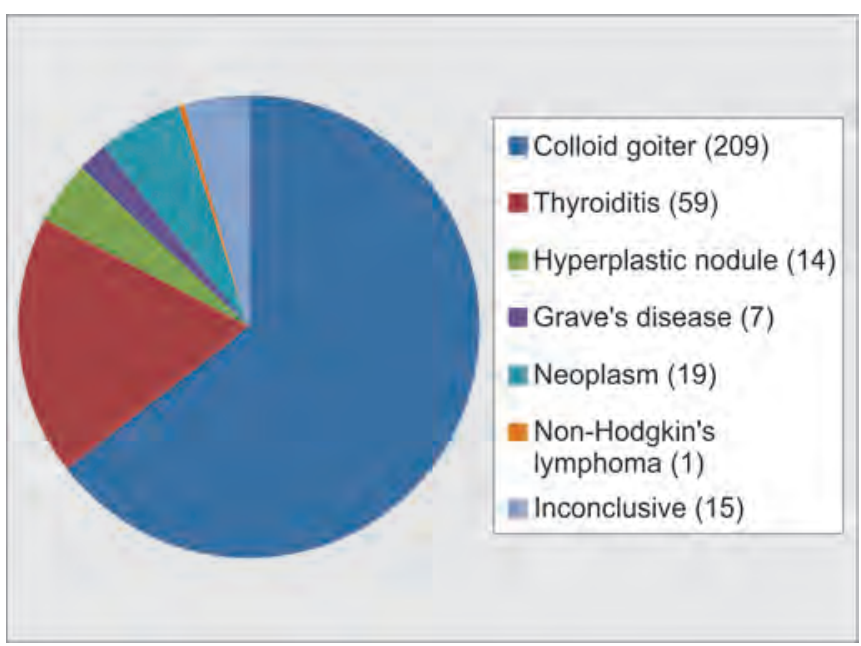

Graph 8: Distribution of thyroid swellings $(n=324)$ 
There were two sites in the oral cavity that were evaluated by FNAC. Hard palate swelling revealed acute inflammatory lesion and floor of mouth swelling was found to be NHL. Both patients were male and in the age 40 to 50 years. Both patients complained of pain and difficulty in swallowing.

\section{DISCUSSION}

This study was carried out to assess the relative frequencies of various pathologies, as assessed by FNAC, occurring as palpable swellings in the head and neck region in those cases who came to our ENT outpatient department. Stewart's opinion of this method still holds good as it was in 1933, when he quoted "diagnosis by aspiration is as reliable as the combined intelligence of the clinician and the pathologist make it." ${ }^{\prime 2}$ Most head and neck masses of distinctive cause present in fairly predictable locations within specific age groups. This permits a methodical approach to clinch a working diagnosis and a differential diagnosis and appropriate treatment protocol for the patient presenting with palpable mass. The patient's age must be an important consideration in the diagnosis. Each age group demonstrates a definite relative frequency of pathologic processes, which can steer the clinician to further differential considerations. The location of a neck swelling is another parameter, i.e., definitely important in the differential diagnosis. Congenital, developmental, and traumatic swellings are fairly predictable in their locations. For the neoplastic pathology, its location is both diagnostically and prognostically important, because in an unviolated neck, the lymphatic spread of head and neck mucosal malignancy and inflammatory processes exhibits a fairly predictable and systematic pattern. ${ }^{3}$ Different etiopathologic processes present with onset of symptoms that vary seemingly in duration. In the literature, a "rule of 7 " has been described, in which average span of clinical features for neck swellings due to infection was 7 days, for neoplasms 7 months, and 7 years was peculiar of developmental pathologies. This rule of 7 was cited in a study spanning 10 years published few decades back. ${ }^{4}$ In our study, maximum number of aspirates was from lymph nodes (53.54\%), followed by thyroid gland $(25.51 \%)$, parotid and submandibular area (5.83 and $2.83 \%$ respectively), and neck (5.51\%), correlating well with other studies. ${ }^{5,6}$

Among all palpable head and neck masses aspirated, lymph node involvement was most common (53.54\%), similarly as observed by others. ${ }^{6,7}$ Cervical lymphadenopathy is a common presentation in adults as well as children, and it usually occurs due to various etiological factors whose frequency depends upon geographical distribution and socioeconomic factors. Familiarity about the pattern of lymph node enlargement in a population studied helps bring about the required cytopathological assessment and assists the clinician in choosing selective and required investigations and treatment plan accordingly. In this group, male to female ratio was 1.27:1, with male preponderance. Swellings, which were reactive in nature $263(38.7 \%)$, dominated our study, and it correlated well with observations of other workers. ${ }^{8,9}$ Tubercular $175(25.7 \%)$, acute suppurative pathology $34(5 \%)$, chronic granulomatous picture $92(13.5 \%)$, and metastatic lymphadenopathy $69(10.2 \%)$ were other significant cytological patterns of lymphadenopathy, whereas Shakya et $\mathrm{al}^{10}$ reported that 50.4, 22.4, 4.8, and 10\% affected lymph nodes were involved in reactive, tubercular, metastatic, and granuloma respectively. In our series, the majority of cases were of benign nature, while $13.38 \%$ cases were malignant. Steel et $\mathrm{al}^{11}$ cited $59 \%$ cases of malignant pathology and $34 \%$ cases of benign pathology in their series. This may be ascribed to the observation that in western world, there is preponderance of malignant and congenital conditions, whereas in developing countries, majority of cases are of reactive, tuberculous, and granulomatous pathology.

Tuberculous infection was a significant differential diagnostic pathology observed (25.7\%), close to as observed by Tilak et al. ${ }^{2}$ Tuberculosis is widely prevalent in developing countries, with prevalence as high as $1.5 \%$, and tubercular cervical lymphadenopathy is the most common form of extrapulmonary tuberculosis (accounting for 30 to $40 \%$ of cases). ${ }^{12}$ Fine needle aspiration cytology has been reported to have a 90 to $100 \%$ diagnostic accuracy in the detection of tuberculous affliction of nodes. ${ }^{13}$ The most confirmatory battery of investigations in establishing cervical tubercular infection, particularly in the context of developing countries, must include culture, cytological analysis of needle aspirates with ZN staining, chest radiography, and Mantoux test coupled with adequate, careful history and thorough clinical examination. Culture of the lymph node aspirate for the organism is necessary if the smears are Bacilli-negative and cytologically uncertain. ${ }^{2}$ Cytological picture of tubercular lymphadenitis may mimic reactive hyperplasia due to viral or toxoplasma infection, particularly in children, where aspirate shows polymorphous picture with occasional epithelioid cells, absence of Langhans giant cells, or caseous necrosis, necessitating open biopsy for confirmation. ${ }^{13}$ As tubercular lymphadenitis may be the sole manifestation of the disease and associated constitutional features may be conspicuous by its absence, it is wise to explore each avenue of history, physical examination coupled with radiological and laboratory investigations for specific facts concerning the diagnosis of tubercular infection. Female preponderance in tubercular infection 
has been reported, ${ }^{12}$ and almost equal predominance $(50.86 \%)$ was observed in our series.

The diagnostic accuracy of cytological diagnosis and classification of lymphoma on FNA sampling varies between 10 and $90 \%{ }^{2}$ In our series, there were a total of 24 cases of lymphoma (1.88\%) (19 NHL, 5 HL cases), whereas others ${ }^{2,8}$ have reported an incidence of 5.6 and $9.5 \%$ in their series respectively. It is often difficult cytologically to differentiate between histiocytic lymphoma and metastatic poorly differentiated nasopharyngeal carcinoma. ${ }^{14}$ Often NHL can be misdiagnosed as reactive lymphadenitis due to mixed population of lymphoid tissue and tingible body macrophage and very large germinal centers. $^{2}$ The definitive diagnosis of lymphoma and its classification must be made on an adequate tissue biopsy. ${ }^{2}$

Cheng and Dorman ${ }^{15}$ in their series of 187 patients, in Auckland, New Zealand, found malignancy to be the causative factor in 50\% of cases. Likewise, Schelkun and Grundy ${ }^{16}$ in Chicago (US) and Schwarz et al $^{17}$ in Columbia (Canada) found malignancy as the major cause of lymphadenopathy ( 40 and $48 \%$ respectively). In our series, secondary metastases in nodes were found in 69 cases $(10.15 \%)$, and majority of them were squamous cell carcinoma, followed by adenocarcinoma, similarly as observed in other studies. ${ }^{6,18,19}$ Smears abundant with inflammatory cells and abscess formation should be circumspectly observed for malignant squamous cells. ${ }^{6}$ Squamous metaplasia in various benign conditions can pretend as well-differentiated squamous cell carcinoma on FNAC. ${ }^{6}$ On correlating pattern of malignant lymphadenopathy with age groups, it was revealed that $27.54 \%$ of cases were in the 7 th decade.

The differential diagnoses of palpable benign cystic lesions in the head and neck region include cases of developmental and acquired causes. Epidermoid cysts are benign lesions histologically, traditionally occurring most commonly in the face, scalp, neck, and trunk, characterized by cystic spaces lined by simple squamous epithelium (epidermoid cyst), containing skin adnexa (dermoid cyst) or tissue of all three germ cell layers (teratoid cyst), and they constitute 1.6 to $6.9 \%$ of all cysts in the head and neck region. ${ }^{20}$ In our series, there were a total of 33 epidermoid cysts occurring prominently in the neck ${ }^{17}$ and face ${ }^{14}$ region. Eight cases of thyroglossal cyst and seven cases of branchial cyst were found in our series. Thyroglossal cyst presents clinically in children, but lesions can also be found in adults. Thyroglossal cyst and branchial cyst may usually present for the first time as infected neck swellings. Fifteen cases of lipoma, two paraganglioma, one spindle cell lesion, and neurofibroma each were found among neck region masses in our series. Cystic lymphangiomas are most commonly observed in the head and neck (75\%) region with a left-sided predilection, while about $20 \%$ occur in the axilla. ${ }^{21}$ In our series, there were total three such swellings, and two of them were left sided. In this (neck and postauricular area) group, 15 cases of lipoma, 2 paraganglioma, 1 spindle cell lesion and neurofibroma each, and 6 benign vascular lesions were identified. Fine needle aspiration cytology mostly encounters no problem in differentiating high-grade soft tissue sarcomas from benign masses, but borderline and low-grade lesions pose diagnostic dilemma. ${ }^{13}$ Metastatic well-differentiated squamous cell carcinoma with tumor necrosis may pose diagnostic dilemma in distinguishing from infected branchial cyst. ${ }^{22}$

Thyroid enlargement is an oft-encountered clinical entity in various regions of the world, and India has the world's biggest goiter belt in the sub-Himalayan territory, ${ }^{23}$ and our study, too, represented that area. Fine needle aspiration cytology is a simple, safe, quick, costeffective diagnostic modality and is a valuable adjunct in preoperative screening in the diagnosis of thyroid masses and an important tool in managing such cases. In a study, colloid goiter was the most common pathology observed (42.2\%), and out of malignant cases, papillary carcinoma represented $71.4 \%$, whereas follicular carcinoma represented $28.6 \%{ }^{24}$ In our study, colloid goiter represented $64.5 \%$ of total lesions, whereas among malignant cases, follicular carcinoma was most common (52.6\%), followed by papillary carcinoma $(21.1 \%)$, Hurthle cell neoplasm (15.8\%), and medullary carcinoma (10.5\%). Cystic thyroid masses often set diagnostic pitfalls. In mixed, solid-cystic lesions or smaller lesions, ultrasound-guided aspiration method is more fruitful than palpation-guided aspiration. Cystic change and/or hemorrhage in neoplasms is observed in a study in about $25 \%$ of papillary carcinomas, $20 \%$ of follicular neoplasms, and $26 \%$ of follicular carcinomas. ${ }^{2}$ In FNAC, there lies a diagnostic trap in differentiating follicular adenoma from carcinoma because capsular/vascular invasion cannot be correctly identified in the smears. The reasons behind false-negative results cited are poor cellular sample in a large cystic papillary carcinoma and the thick fibrous capsule. ${ }^{13}$ Similarly, differentiating parathyroid neoplasm and follicular neoplasm of thyroid cytologically can sometimes be difficult. ${ }^{2}$ Marked cellularity of the cytological specimen is another problem, quite natural, in thyroid aspiration cytology. Increased cellularity and loss of cohesion may be present in hyperplastic/adenomatous goiter, adenoma, or in carcinoma. ${ }^{23}$ Multiple aspirations on each palpable mass in a thyroid swelling and for each rapidly changing diffuse lesion of thyroid are advisable to avoid erroneous results and also due to the presence of more than one pathologic processes, in thyroid, at a time. ${ }^{2}$ Importance of FNAC, in the context of thyroid lesion, lies in the fact that certain lesions like thyroiditis and colloid goiter can be managed 
conservatively, whereas lymphoma and undifferentiated carcinoma of thyroid can be treated with radiotherapy or chemotherapy, thus obviating the need of surgical intervention in such cases.

Evaluation of suspected salivary gland lesions demands a systematic rational approach. Establishing whether the lesion is truly of salivary origin or not is of paramount importance. Eventually, identification of cellular and morphological architecture to classify them into cystic, inflammatory, or neoplastic categories substantially obviates unnecessary surgery in a significant number of cases. Among the salivary swellings, parotid was the most commonly involved gland (63.24\%). Benign tumors accounted for $84.31 \%$ of total neoplasms. Parotid was the most common site for both benign $(88.37 \%)$ and malignant tumors ( $87.5 \%)$. A total of 18 out of 43 benign neoplasms occurred in females (female:male $=0.72: 1$ ), whereas 5 out of 8 malignancies occurred in females (female:male $=1.67: 1)$. Similar observations have also been reported. ${ }^{25}$ Among benign tumors (submandibular and parotid area), pleomorphic adenoma (34 cases) was the commonest pathology, while mucoepidermoid carcinoma was the next most common malignant pathology (3 cases) observed, similar to findings reported by others. ${ }^{13,26}$ It was also observed that the most common pathology observed in submandibular gland was sialadenitis, followed by pleomorphic adenoma and retention cyst, while the most common pathology found in parotid gland was pleomorphic adenoma, followed by sialadenitis. Low-grade mucoepidermoid carcinoma and pleomorphic adenoma need to be individualized and interpreted carefully as they usually pose a diagnostic dilemma. ${ }^{27}$ The most important reasons leading to misdiagnosis and false negatives are lack of necessary expertise, presence of atypical cells in both benign and malignant lesions, association of chronic sialadenitis with several types of malignancy, and difficulty in diagnosing lymphoma. ${ }^{28}$ Diagnostic dilemma has been reported in cases where mucoepidermoid carcinoma and/or Warthin's tumor present as cystic swellings, aspiration reveals inflammation instead of pleomorphic adenoma, adenoid cystic carcinoma is misinterpreted as pleomorphic adenoma or basal cell adenoma on aspiration cytology, and Warthin's tumor is cytodiagnosed as squamous cell carcinoma. ${ }^{25}$

There are various lesions in the oral cavity, floor of mouth, tongue, palate, tonsils, and posterior pharyngeal wall that can be assessed by needle under visual control; however, little data have been published regarding usefulness of FNAC in the diagnosis of intraoral lesions. ${ }^{29,30}$ Dejmek and Lindholm ${ }^{31}$ cited $85 \%$ diagnostic accuracy of FNAC for cystic and solid lesions of the oral cavity, whereas Gunhan et $\mathrm{al}^{29}$ reported diagnostic accuracy of 92 and $97 \%$ for malignant and benign lesions respectively, of the oral cavity and jaw. Cramer et $a l^{30}$ described the usefulness of FNAC in evaluation of parapharyngeal, palatal masses, and submucosal lesions of the oral cavity, while Domanski and Akerman $^{32}$ suggested this technique as the initial diagnostic step in assessing tongue swellings.

However, FNAC has certain limitations. It requires a skilled cytologist for interpreting slides correctly. It also requires much effort while dealing with small lesions, which tend to slip away. The incidence of inadequacy or inconclusiveness in various reports has ranged from 0 to $25 \%{ }^{13}$ and 3 to $30 \% .{ }^{5}$ Fine needle aspiration cytology gives information regarding cellular architecture but it lacks histological subtyping. Accuracy of aspiration cytology depends upon location and pathologic type of mass, expertise, sample adequacy, endemicity, methods used to aid in diagnosis, and age of the patient. ${ }^{6}$ Tilak et $\mathrm{al}^{2}$ highlighted the limitations of this technique while dealing with head and neck swellings:

- Difficulty in diagnosing and subclassifying lymphomas.

- Differentiating colloid goiter from follicular adenoma.

- Isolating colloid goiter from macrofollicular papillary carcinomas.

- Distinguishing thyroid adenoma from early follicular carcinomas.

- Typing the different reactive lymphadenopathies. ${ }^{13}$

- To categorize the borderline and malignant soft tissue tumors. ${ }^{13}$

\section{CONCLUSION}

Fine needle aspiration cytology is a quick, convenient, easy to perform, and fairly accurate method of cytological diagnosis, management, and follow-up that can be exercised on an outpatient basis. Importance of this study, particularly in the context of parotid masses, lies in the fact that a preoperative cytological diagnosis of a benign lesion in a high-risk patient may obviate the need of a surgical intervention. Preoperative diagnosis of certain conditions like lymphoma and inflammatory pathology may also escape from unnecessary surgeries. This procedure is effectively safe, free from complications, and usually does not require anesthetic protocols, therefore, producing speedy results.

\section{REFERENCES}

1. Ramzy, I. Aspiration biopsy techniques. In: Ramzy, I., editor. Clinical cytopathology and aspiration biopsy-fundamental principles and practice. 2nd ed. Hong Kong: McGraw-Hill; 2001. p. 347.

2. Tilak V, Dhaded AV, Jain R. Fine needle aspiration cytology of head and neck masses. Indian J Pathol Microbiol 2002 Jan;45(1):23-30.

3. McGuirt, W.F. Neck masses: differential diagnosis and therapeutic approach. In: Shockley, W.W.; Pillsbury, H.C., editors. 
The neck diagnosis and surgery. St. Louis, MO: Mosby-Year Book; 1994. p. 73.

4. Solem BS, Schroder KE, Mair IWS. Differential diagnosis of a mass in the upper lateral neck. J Laryngol Otol 1981 Oct;95(10):1041-1047.

5. Tandon S, Shahab R, Benton JI, Ghosh SK, Sheard J, Jones TM. Fine needle aspiration cytology in a regional head and neck cancer center: comparison with a systematic review and meta-analysis. Head Neck 2008 Sep;30(9): 1246-1252.

6. Modi P, Oza H, Bhalodia J. Utility and adequacy of fine needle aspiration cytology in head and neck lesions: a hospital based study. Int J Sci Stud 2014;2(8):100-105.

7. Singal P, Bal MS, Kharbanda JS, Sethi PS. Efficacy of fine needle aspiration in head and neck lesions. Int J Med Dent Sci 2014;3:421-430.

8. Egea AS, Gonzalez MAM, Pérez Barrios A, Alberti Masgrau $\mathrm{N}$, de Agustín de Agustín P. Usefulness of light microscopy in lymph node fine needle aspiration biopsy. Acta Cytol 2002 Mar-Apr;46(2):368-369.

9. El Hag IA, Chiedozi LC, Al Rayees FA, Kollur SM. Fine needle aspiration cytology of head and neck masses. Seven years' experience in a secondary care hospital. Acta Cytol 2003 May-Jun;47(3):387-392.

10. Shakya G, Malla S, Shakya KN, Shrestha R. A study of FNAC of cervical lymph nodes. J Nepal Health Res Council 2009;7(1):1-5

11. Steel BL, Schwartz MR, Ibrahim R. Fine needle aspiration biopsy in diagnosis of lymphadenopathy in 1103 patients. Acta Cytol 1995 Jan-Feb;39(1):76-81.

12. Khan RA, Wahab S, Chana RS, Naseem S, Siddique S. Children with significant cervical lymphadenopathy: clinicopathological analysis and role of fine needle aspiration in Indian setup. J Pediatr (Rio J) 2008 Sep-Oct;84(5): 449-454.

13. Fernandes H, D'souza CRS, Thejaswini BN. The role of fine needle aspiration cytology in palpable head and neck masses. J Clin Diagn Res 2009;3:1719-1725.

14. Mondal A, Roychaudhuri BK. FNAC in diagnosis of head and neck tumours: a study of 1082 cases. Ind J Otolaryngol Head Neck Surg 1992;1(4):176-180.

15. Cheng AT, Dorman B. Fine needle aspiration cytology: the Auckland experience. Aust N Z J Surg 1992 May;62(5): 368-372.

16. Schelkun PM, Grundy WG. Fine needle aspiration biopsy of head and neck lesions. J Oral Maxillofac Surg 1991 Mar;49(3):262-267.
17. Schwarz R, Chan NH, Macfarlane JK. Fine needle aspiration cytology in the evaluation of head and neck masses. Am J Surg 1990 May;159(5):482-485.

18. Hajdu SI, Melamed MR. The diagnostic value of aspiration smears. Am J Clin Path 1973 Mar;59(3):350-354.

19. Engzell V, Jakobsson PA, Sigurdson A, Zajidek. Aspiration biopsy of metastatic carcinoma in lymph node of neck. Acta Otolaryngol 1971 Jul-Aug;72(1):138-147.

20. Dutta M, Saha J, Biswas G, Chattopadhyay S, Sen I, Sinha $R$. Epidermoid cysts in head and neck: our experience with review of literature. Indian J Otolaryngol Head Neck Surg 2013 Jul;65(Suppl 1):14-21.

21. Acevedo JL, Shah RK, Neville HL, Poole MD. Cystic hygroma http://emedicine.medscape.com/article/994055-overview. Accessed on 5 Feb 2015.

22. Pontiflex AH, Roberts FJ. Fine needle aspiration biopsy cytology in diagnosis of inflammatory lesions. Acta Cytol 1985 Nov-Dec;29(6):979-982.

23. Handa U, Garg S, Mohan H, Nagarkar N. Role of fine needle aspiration cytology in diagnosis and management of thyroid lesions: a study on 434 patients. J Cytol 2008;25(1):13-17.

24. Rout K, Ray CS, Behera SK, Biswal R. A comparative study of FNAC and histopathology of thyroid swellings. Indian J Otolaryngol Head Neck Surg 2011 Oct;63(4):370-372.

25. Sengupta S, Roy A, Mallick MG, Kundu B, Das SK, Das S. FNAC of salivary glands. Indian J Otolaryngol Head Neck Surg 2002 Jul;54(3):184-188.

26. Fernandes GC, Pandit AA. Diagnosis of salivary gland tumours by FNAC. Bombay Hosp J 2000;42(1):1-5.

27. Kotwal M, Gaikwad S, Patil R, Munshi M, Bobhate S. FNAC of salivary gland - a useful tool in preoperative diagnosis or a cytopathologist's riddle? J Cytol 2007;24(2):85-88.

28. Javadi M, Asghari A, Hassannia F. Value of Fine-needle aspiration cytology in the evaluation of parotid tumours. Indian J Otolaryngol Head Neck Surg 2012 Sep:64(3):257-260.

29. Gunhan O, Dogan N, Celasun B, Sengun O, Onder T, Finci R. Fine needle aspiration cytology of oral cavity and jaw bone lesions. A report of 102 cases. Acta Cytol 1993 Mar-Apr;37(2):135-141.

30. Cramer H, Lampe H, Downung P. Intraoral and transoral fine needle aspiration. A review of 25 cases. Acta Cytol 1995 Jul-Aug;39(4):683-688.

31. Dejmek A, Lindholm K. Fine needle aspiration biopsy of cystic lesions of the head and neck, excluding the thyroid. Acta Cytol 1990 May-Jun;34(3):443-448.

32. Domanski HA, Akerman M. Fine needle aspiration cytology of tongue swellings: a study of 75 cases. Diagn Cytopathol 1998 Sep;19(3):229-234. 